\title{
Reproduction of slow-drift motions of a floating wind turbine using second-order hydrodynamics and Operational Modal Analysis
}

\author{
Pegalajar Jurado, Antonio Manuel; Bredmose, Henrik
}

Published in:

Marine Structures

Link to article, DOI:

10.1016/j.marstruc.2019.02.008

Publication date:

2019

Document Version

Peer reviewed version

Link back to DTU Orbit

Citation (APA):

Pegalajar Jurado, A. M., \& Bredmose, H. (2019). Reproduction of slow-drift motions of a floating wind turbine using second-order hydrodynamics and Operational Modal Analysis. Marine Structures, 66, 178-196.

https://doi.org/10.1016/..marstruc.2019.02.008

\section{General rights}

Copyright and moral rights for the publications made accessible in the public portal are retained by the authors and/or other copyright owners and it is a condition of accessing publications that users recognise and abide by the legal requirements associated with these rights.

- Users may download and print one copy of any publication from the public portal for the purpose of private study or research.

- You may not further distribute the material or use it for any profit-making activity or commercial gain

- You may freely distribute the URL identifying the publication in the public portal 


\title{
Reproduction of slow-drift motions of a floating wind turbine using second-order hydrodynamics and Operational Modal Analysis
}

\author{
Antonio Pegalajar-Jurado and Henrik Bredmose \\ Department of Wind Energy, Technical University of Denmark, Nils Koppels Allé, building \\ 403, DK-2800 Kgs. Lyngby, Denmark
}

\begin{abstract}
Slow-drift forcing and damping are important for an accurate reproduction of the low-frequency motion of floating offshore wind turbines. In this study we present a numerical model that includes both inviscid slow-drift forcing through full quadratic transfer functions (QTFs) and viscous forcing. To accommodate a linear damping matrix that represents all the damping effects on the floater, the classical Morison formulation of the drag forcing with relative velocity is simplified into a pure forcing term and a linear constant damping term. The frequency-dependent radiation properties are also replaced by constant matrices. We investigate the application of Operational Modal Analysis (OMA) to estimate the damping ratios from the test data, and also pursue a calibration method where the linear damping is calibrated in the modal space and subsequently transformed back to the physical space. The model is compared to wave basin data for four environmental conditions including the 50-year sea state. The damping ratios estimated with OMA generally increase with the sea state. The measured response can be generally well reproduced by the model when the damping is calibrated for each sea state. Due to the neglection of firstorder motion effects in the applied QTFs and to the damping introduced by the mooring lines, however, the surge response is underpredicted for the severe sea states, and the yaw motion is always underpredicted. The commonly used approach where the damping is calibrated to the decay tests is found to provide less accurate results than the approach of calibrating the modal damping ratios
\end{abstract}


for the given sea state. The damping ratios estimated with OMA are found similar to the calibrated values for mild pink-noise sea states, while significant differences exist for the stronger Pierson-Moskowitz sea states. Thus the potential and limitations for OMA application in different sea states is discussed. In summary, the model is found able to generally predict the measured response, provided that the damping is properly calibrated for each sea state.

Keywords: Floating substructure, slow drift, nonlinear hydrodynamics, damping, Operational Modal Analysis

2010 MSC: 00-01, 99-00

\section{Introduction}

The interest in floating offshore wind energy has grown significantly in recent years, with spar buoys and semisubmersibles as the most popular concepts. Being heavy marine structures restrained by soft mooring systems, these floaters

5 typically have very low natural frequencies for the horizontal degrees of freedom (DoFs), namely surge, sway and yaw. Although the floating structures are designed to avoid resonance with the linear spectrum of wave loads, second-order slow-drift loads can excite the motion of the low-frequency horizontal modes, which combined with low damping levels can lead to resonance and high response levels for the mooring system. Therefore, slow-drift loads and response play an important role in the design of mooring systems for floating offshore structures. Viscous effects induce both forcing and damping, thus they are crucial to correctly estimate the slow-drift resonant response of the floating system. While consensus exists on how to include slow-drift loads in the numerical models, viscous effects are sometimes neglected, both with respect to forcing and damping. In other cases the damping coefficients are chosen to reproduce decay tests in still water or manually calibrated to yield the desired response. The aim of the present paper is to numerically reproduce the slow-drift response observed in physical tests, by combining both viscous and inviscid slow-drift forcing with a simplified damping model, suitable for efficient solution of the re- 
sponse. We further investigate the applicability of Operational Modal Analysis to extract damping levels from the physical model tests of a semi-submersible floating wind turbine in operational sea states of Thys et al. 1].

A number of studies exist in the literature that address numerical modelling 25 of second-order hydrodynamic loads on floating structures. Roald et al. [2] investigated second-order effects on a spar and a tension-leg platform (TLP), by comparing first- and second-order response-amplitude operators (RAOs) in the frequency domain. The numerical model of the floating wind turbine was linearized and the system matrices were applied to compute the full quadratic transfer functions (QTFs) and RAOs. Viscous effects were not represented in the study. A similar approach was followed by Bayati et al. 3] to analyze the effect of second-order hydrodynamics on a semisub, also computing full QTFs and neglecting viscous forcing and damping in the frequency-domain analysis.

A time-domain analysis — which allows the inclusion of nonlinear effects such as viscous drag and catenary mooring loads - of the second-order hydrodynamic loads on a spar was carried out by Duarte et al. 4. They validated the implementation of second-order loads in FAST [5] by comparison to OrcaFlex [6. Results were compared for ten-minute simulations using both full QTFs and the Newman approximation (Newman [7, Standing et al. [8]), and significant 40 differences between the two approaches were found. They also concluded that simulation times longer than ten minutes are needed to fully capture the effects of slow-drift loads, and that the sum-frequency loads did not have a significant contribution to the response of the floater considered. Viscous effects and their influence on second-order response was not considered in their work. A similar validation study was carried out by Gueydon et al. 9], who investigated the effect of second-order loads on a semisub using two different numerical codes. The viscous damping was represented with a quadratic damping matrix and with an additional linear damping coefficient tuned to match the surge drift motion. They identified the resonant behaviour caused by second-order loads and acknowledged the important role that viscous damping plays. They further concluded that sum-frequency loads had negligible effects on the motion of the 
floater analyzed.

The numerical modelling of slow-drift motions has been compared to test data by, for example, Coulling et al. [10. A FAST model of a semisub floating wind turbine was used, including second-order loads only in surge computed with Newman's approximation. The model, which did not include Morison drag, accounted for viscous effects through quadratic damping coefficients, tuned to match decay tests in still water and later reduced to match the observed response in waves. They found that, although including second-order loads in the model significantly improved the prediction of the surge motion, the response was driven by resonance and was therefore highly dependent on the damping included in the model.

Response to second-order wave loads has been investigated intensively also in the oil and gas industry. In the comprehensive study of Matos et al. [11, ${ }_{65}$ they simulated and compared to physical tests the resonant heave, roll and pitch motions obtained through a WAMIT 12 analysis with different modelling alternatives: Newman's approximation; full QTFs; full QTFs neglecting the freesurface forcing; and QTFs neglecting the contribution of the second-order velocity potential. They also investigated the so-called "white-noise approach", which consists on computing only certain bands of the QTF matrices - corresponding to difference frequencies close or equal to the natural frequencies of the floater - to reduce the computational effort required. Their numerical response was based on a linear frequency-domain model including linearized mooring stiffness and linear viscous damping, where the latter was tuned to match the observed response. Among their findings, based on power spectral density (PSD) plots, was that both Newman's approximation and using the QTFs without the contribution of the second-order potential did not give accurate results. On the other hand, full QTFs neglecting the free-surface forcing and the "white-noise approach" provided satisfactory results when compared to test data. Simos et so al. [13] presented a study similar to [11, but applied to a semisub for floating offshore wind application where the motion of interest was surge. In their frequency-domain model two damping levels were considered, corresponding to 
the limits of the damping range observed for decay tests in still water. They recognized the importance of damping in the estimation of the resonant motion, and the uncertainty introduced by the choice of damping. Their conclusions, similar to those of [11, stated that while Newman's approximation led to an underestimation of slow-drift motions, neglecting the free-surface forcing and the second-order potential in the QTFs gave good results, as did the "white-noise approach".

While the above comparisons to test data provide valuable expertise on the modelling of slow-drift hydrodynamic loads, they neglect viscous forcing and represent viscous damping by a linear damping term calibrated to match the desired response. Yet, some of them agree on the importance of viscous effects to correctly predict slow-drift response. Lemmer et al. 14 compared a loworder model that included slow-drift loads through the Newman approximation and Morison drag based on the relative floater velocity to test data. The drag coefficients were tuned for different sea states to match the measured response, given that the values tuned to the decay tests did not reproduce well the response in waves. A similar study was carried out by Kvittem et al. [15, where linear damping coefficients in surge and sway together with Morison drag using depth-dependent drag coefficients provided a good match of the measured response. They also found that different drag coefficients were needed for different sea states, and acknowledged that both damping and forcing are altered when changing such values. They reported difficulties matching the response in all DoFs simultaneously, and worse results when the Morison drag term was replaced by a quadratic damping matrix.

It is clear in the literature that, since the local viscous damping is proportional to the fluid velocity and thus increases with the sea state severity, the damping parameters calibrated for decay tests cannot be expected to work for all sea states. Hence, a more systematic approach to include damping in the numerical models is explored in the present study. We apply a method where we extract the damping information directly from the test data and apply it within the numerical model. The extraction of damping is done through Operational 
Modal Analysis (OMA), a set of methods that provide modal properties of a structure - usually natural frequencies, damping ratios and mode shapes through the analysis of response data in operational conditions. Since the OMA toolset is output-only (meaning that only the measured structural response is needed), it has the potential to become a robust and model-independent way of estimating the modal properties of floating wind turbines. Although OMA comprises several different methods, they all work under the same set of assumptions: i) the system can be described with linear equations of motion; ii) the motions are lightly damped; and iii) the excitation loads can be considered as white noise. While (i) and (ii) are reasonably fulfilled for floating offshore wind turbines in wave forcing, (iii) is questionable since the low-frequency modes are forced by second-order subharmonic waves from a usually narrow-banded linear wave spectrum. A central aspect of the present investigation is thus how well OMA works for damping detection of floating wind turbines, and to which extent this is influenced by especially assumption (iii).

Recently, OMA techniques have been applied to bottom-fixed offshore wind turbines. In Pahn et al. [16] the inverse load calculation method was applied to a real offshore monopile wind turbine, where the Frequency Domain Decomposition (FDD) method was employed to extract dynamic system information such as inertia, stiffness and damping characteristics. Bajrić et al. [17] applied three different OMA methods - Eigensystem Realization Algorithm (ERA), covariance-driven Stochastic Subspace Identification (COV-SSI) and Enhanced Frequency Domain Decomposition (EFDD) - to estimate the damping of tower vibrations from measurements of a monopile wind turbine. One of their conclusions was that discrepancies in the estimated damping can arise when closely spaced modes are present (such as fore-aft and side-side tower bending modes with idling or parked wind turbine).

The application of OMA techniques to the rigid-body motion of floating wind turbines was first done by Ruzzo et al. [18. In their work, which was purely numerical, a numerical model was set up for a spar floating wind turbine, with Morison-based hydrodynamics and linear waves from a JONSWAP spectrum 
1. that the quadratic damping contribution in the Morison drag dominates for decay tests in still water, while the linear damping term becomes more important in waves; 
3. the wish for easy calibration; and

4. compatibility with OMA results.

We explore OMA as a tool to estimate the linear damping matrix for different wave conditions, and also investigate further simplifications of the hydrodynamic shapes. The OMA methods work under the following assumptions: i) the system behaves in a linear manner; ii) the motions are lightly damped, with a damping ratio $\zeta$ between $10-15 \%$; and iii) the excitation loads can be considered as white 
noise in the frequency band of interest. Many of these methods are summarized in Brincker and Ventura 23. For this study, the Ibrahim Time Domain (ITD) method [24, 25, 26] was selected, and it is briefly introduced here.

The starting point is a matrix $\mathbf{x}(t)$ containing time series of the measured data. If $n t$ samples and $n c$ channels are available, $\mathbf{x}(t)$ has $n c$ rows and $n t$ columns. For each time lag $\tau$, a correlation matrix $\mathbf{y}(\tau)$ can be built, where each element is given by

$$
y_{i j}(\tau)=\frac{1}{T} \int_{0}^{T} x_{i}(t) x_{j}(t+\tau) d t
$$

and $T$ is the duration of the time series. The idea behind the ITD method is that any free response - and hence the correlation matrix $\mathbf{y}(t)$ - can be written as a linear combination of mode shapes and exponential decays,

$$
\mathbf{y}(t)=\mathbf{y}(k \Delta t)=c_{1} \mathbf{a}_{1} e^{\lambda_{1} k \Delta t}+c_{2} \mathbf{a}_{2} e^{\lambda_{2} k \Delta t}+\ldots=c_{1} \mathbf{a}_{1} \mu_{1}^{k}+c_{2} \mathbf{a}_{2} \mu_{2}^{k}+\ldots
$$

where $\mathbf{a}_{n}$ are the mode shapes, $\lambda_{n}$ are the continuous time poles, $\mu_{n}$ are the discrete time poles and $c_{n}$ are the initial modal amplitudes given by the correlation matrix at $t=0$,

$$
\mathbf{y}(t=0)=c_{1} \mathbf{a}_{1}+c_{2} \mathbf{a}_{2}+\ldots
$$

Next, a block Hankel matrix $\mathbf{H}$ with four block rows is built, where each block row is the one above delayed one time step $\Delta t$. This matrix $\mathbf{H}$ is split into two smaller matrices $\mathbf{H}_{1}$ and $\mathbf{H}_{2}$ with two block rows each,

$$
\mathbf{H}=\left[\begin{array}{cccc}
\mathbf{y}(1) & \mathbf{y}(2) & \ldots & \mathbf{y}(n t-3) \\
\mathbf{y}(2) & \mathbf{y}(3) & \ldots & \mathbf{y}(n t-2) \\
\mathbf{y}(3) & \mathbf{y}(4) & \ldots & \mathbf{y}(n t-1) \\
\mathbf{y}(4) & \mathbf{y}(5) & \ldots & \mathbf{y}(n t)
\end{array}\right]=\left[\begin{array}{l}
\mathbf{H}_{1} \\
\mathbf{H}_{2}
\end{array}\right] .
$$

Considering Eqn. (2), $\mathbf{H}_{1}$ can be written as

$$
\mathbf{H}_{1}=\mathbf{\Psi} \boldsymbol{\Lambda}
$$


where the columns of $\boldsymbol{\Psi}$ contain the mode shapes and $\boldsymbol{\Lambda}$ contains the discrete time poles and modal amplitudes. In a similar way, $\mathbf{H}_{2}$ can be expressed as

$$
\mathbf{H}_{2}=\boldsymbol{\Psi}\left[\mu_{n}\right]^{2} \boldsymbol{\Lambda}
$$

since it is $\mathbf{H}_{1}$ delayed two time steps. Here $\left[\mu_{n}\right]$ is a diagonal matrix containing the discrete eigenvalues. Combining Eqns. (5) and (6) $\Lambda$ can be eliminated, 225 resulting in

$$
\boldsymbol{\Psi}^{-1} \mathbf{H}_{1}=\left[\mu_{n}\right]^{-2} \boldsymbol{\Psi}^{-1} \mathbf{H}_{2} \quad \Longrightarrow \quad \mathbf{D H}_{1}=\mathbf{H}_{2} .
$$

Here $\mathbf{D}=\boldsymbol{\Psi}\left[\mu_{n}\right]^{2} \boldsymbol{\Psi}^{-1}$ is the system matrix, which can be estimated as

$$
\hat{\mathbf{D}}=\frac{\mathbf{H}_{2} \mathbf{H}_{1}^{T}\left(\mathbf{H}_{1} \mathbf{H}_{1}^{T}\right)^{-1}+\mathbf{H}_{2} \mathbf{H}_{2}^{T}\left(\mathbf{H}_{1} \mathbf{H}_{2}^{T}\right)^{-1}}{2} .
$$

The final step entails the eigenvalue decomposition of the estimated system matrix $\hat{\mathbf{D}}$, yielding the mode shapes in the columns of $\boldsymbol{\Psi}$ and the squared discrete time poles in the diagonal of $\left[\mu_{n}\right]^{2}$. The continuous time poles $\lambda_{n}$ are found from $\mu_{n}=e^{\lambda_{n} \Delta t}$ and come in pairs of complex conjugates. The modal natural frequencies $\omega_{n}$ and damping ratios $\zeta_{n}$ are given by

$$
\begin{array}{r}
\omega_{n}=\left|\lambda_{n}\right|, \\
\zeta_{n}=-\frac{\Re\left\{\lambda_{n}\right\}}{\omega_{n}},
\end{array}
$$

where $\Re$ refers to the real part.

\section{The case study}

The object of study in the present work is the DTU 10MW Reference Wind 235 Turbine (Bak et al. [27) mounted on the NAUTILUS-10 floating substructure (Galvan-Fernandez et al. 28]). The semi-submersible floater (see Fig. 1) was designed by NAUTILUS Floating Solutions SL (http://www.nautilusfs.com/), and its public version is extensively described in $\mathrm{Yu}$ et al. [29]. 


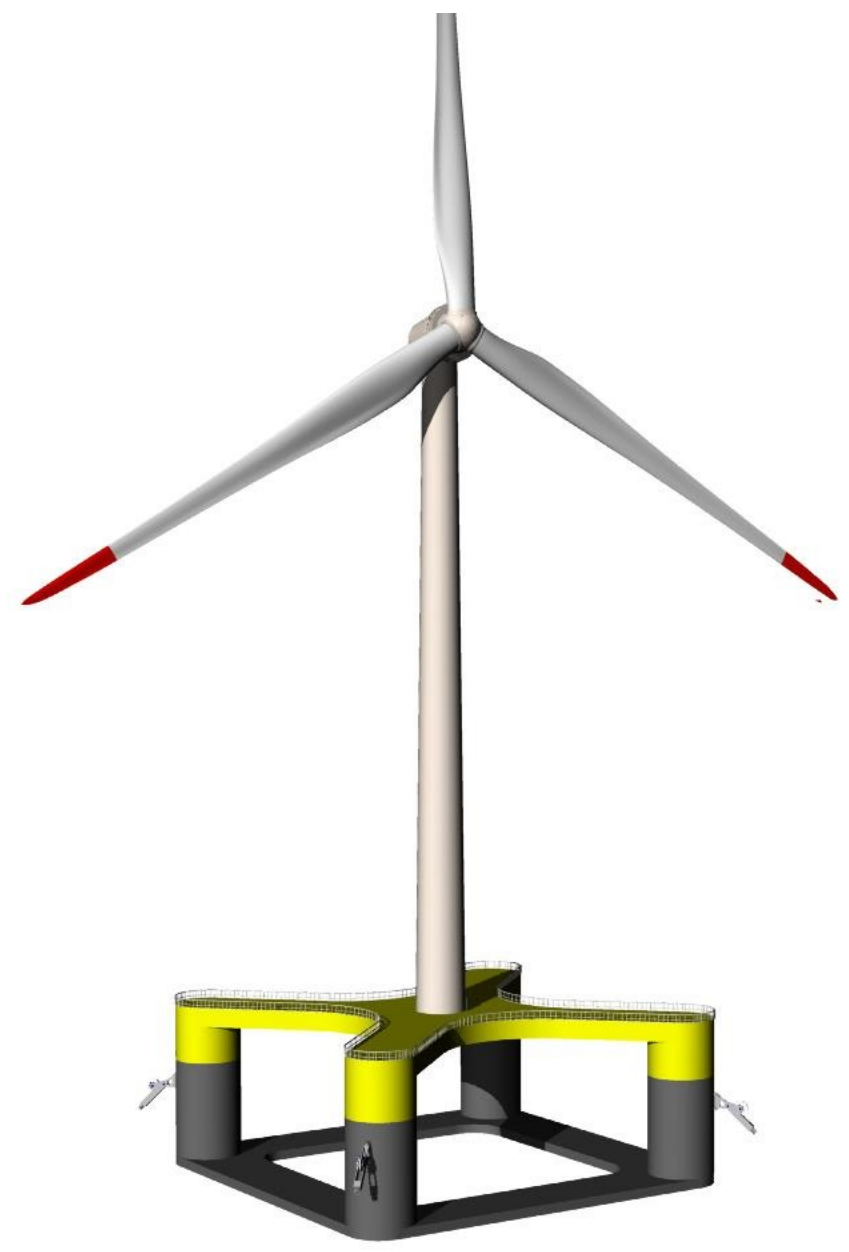

Figure 1: The NAUTILUS-DTU10 floating wind turbine 29.

The NAUTILUS-10 floater consists of four vertical cylindrical columns connected by a square pontoon at the bottom and by an X-shaped deck at the top, where the tower is mounted. The main material is steel, with concrete used as passive ballast and sea water employed for an active ballast system. The floating substructure is moored with four catenary lines. Some of the main properties are collected in Tab. 1 
Table 1: Key figures for the LIFES50+ NAUTILUS-10 floating substructure 29].

\begin{tabular}{cccccr}
$\begin{array}{c}\text { Water } \\
\text { depth }\end{array}$ & $\begin{array}{c}\text { Mooring } \\
\text { length }\end{array}$ & Draft & Freeboard & $\begin{array}{c}\text { Displaced } \\
\text { volume }\end{array}$ & $\begin{array}{r}\text { Mass incl. } \\
\text { ballast }\end{array}$ \\
\hline $130 \mathrm{~m}$ & $833.24 \mathrm{~m}$ & $18.33 \mathrm{~m}$ & $7.67 \mathrm{~m}$ & $9281 \mathrm{~m}^{3}$ & $9337 \mathrm{t}$ \\
\hline
\end{tabular}

\section{The test campaign}

घ The test campaign, carried out at the Sintef Ocean Basin Laboratory (www . sintef.no/en/ocean) in 2018, is described in detail in Thys et al. [1, 30]. Froude scaling with a geometric scale factor of 1:36 was applied. The data, as well as the results presented in this study, are given in full scale unless stated otherwise. The lab-scale floating wind turbine (see Fig. 20 consisted of a stiff floater and tower, connected by a flexible spring at the tower base designed to match the first coupled tower natural frequency. Following the hardware-inthe-loop (HIL) testing approach (Sauder et al. [31, Chabaud et al. 32]), the aerodynamic loads computed in real time by a numerical model were exerted on the tower top by a system of actuator cables. The present study, however, focuses on the wave-only cases where the system of cables was not connected. In the laboratory tests, the tested configuration was slightly different relative to the design of Tab. 1. with a draft of $17.36 \mathrm{~m}$, a freeboard of $8.64 \mathrm{~m}$, a total mass (incl. ballast) of $8884 \mathrm{t}$ and a displaced volume of $8946 \mathrm{~m}^{3}$.

60 The instrumentation relevant to this study includes:

- Wave probes to measure free-surface elevation. A wave calibration phase took place before the floating structure was placed in the basin, in which the free-surface elevation was measured at three locations using conductancetype wave probes. One of the wave probes (W2) was placed at the location where the centre of the floating structure would later be. For this study, the raw time series of free-surface elevation measured by the wave probe W2 were used as the input to the numerical model for each sea state. Another wave probe (W1) used in the calibration phase was kept during the 


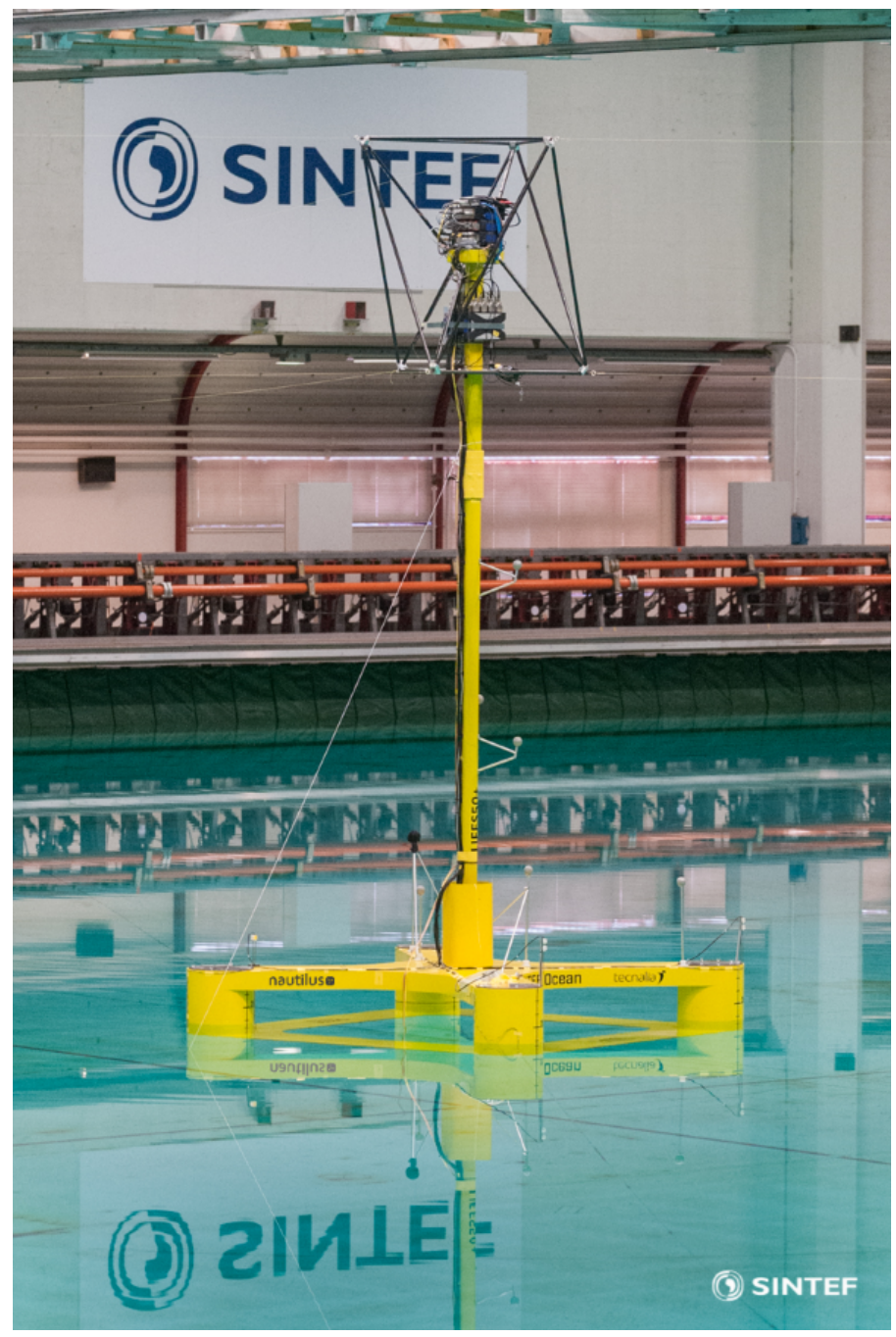

Figure 2: Testing of the NAUTILUS-DTU10 floating wind turbine at Sintef Ocean [1].

tests. It was therefore possible to assess the wave repeatability between the calibration phase and the actual testing phase.

- Force transducers to record mooring line tension at the upper end (fairlead) of each mooring line. The pre-tension was measured once at the start of the test campaign, and deviations in the four lines between $-6.82 \%$ and $-9.46 \%$ when compared to the target are reported in [1]. For each test, the 
force sensors were zeroed before the test and the pre-tension was added afterwards.

- A Qualisys motion capture system to track the floater motion in 6 DoFs. The system consisted of four reflective markers placed on the front part of the floater's top deck, and two infrared cameras located outside of the basin. The motion signals were zeroed once at the start of the test campaign.

The floating wind turbine was placed in the basin 15 deg rotated counterclockwise with respect to the main wave direction. Although different wave headings were tested, this study only considers cases where the waves come from the main direction. Table 2 shows the four environmental conditions considered here, all of them with a total duration of $11180 \mathrm{~s}$, equal to $3 \mathrm{~h}$ and 1080 $\mathrm{s}$ in full scale. In the table, $H_{s}$ indicates the significant wave height of each sea state, while $T$ corresponds to the period range for the pink-noise spectra ( $\mathrm{P} 1$ and P2) and to the spectral peak period for the Pierson-Moskowitz spectra (M1 and M2). The case M2 corresponds to the 50-year sea state for the selected site in the Gulf of Maine (see Krieger et al. [33]).

Table 2: Subset of target environmental conditions in [1] selected for this study.

\begin{tabular}{lccr}
\hline Case & Spectrum & $\boldsymbol{H}_{\boldsymbol{s}}[\mathbf{m}]$ & $\boldsymbol{T}[\mathbf{s}]$ \\
\hline P1 & Pink noise & 2.0 & $4.5-18.2$ \\
P2 & Pink noise & 4.0 & $4.5-18.2$ \\
M1 & Pierson-Moskowitz & 7.7 & 12.4 \\
M2 & Pierson-Moskowitz & 10.9 & 15.0 \\
\hline
\end{tabular}

\section{The numerical model}

The numerical model is an in-house tool specifically developed for the present study. Aerodynamic loads are not included and the rotor is represented by a 
point mass and inertia with the values given in [1]. Further, linked to the focus on low-frequency response, the wind turbine tower is considered rigid. Dynamic mooring loads are included through the MoorDyn model 34 coupled through a dynamic-link library (DLL). The model captures the six rigid-body DoF of a floating wind turbine: surge, sway, heave, roll, pitch and yaw. The level of fidelity is similar to standard state-of-the-art aeroelastic tools, although some simplifications to the standard modelling techniques are introduced, as detailed later in this section.

The starting point for the model are the linearized equations of motion (EoM) of a floating body (Cummins [35, Ogilvie 36]) with forcing terms from waves, mooring reactions and viscous effects,

$$
\left(\mathbf{M}+\mathbf{A}_{\infty}\right) \ddot{\boldsymbol{\xi}}+\int_{0}^{t} \mathbf{K}(t-\tau) \dot{\boldsymbol{\xi}}(\tau) d \tau+\mathbf{C}_{h s t} \boldsymbol{\xi}=\mathbf{F}_{e x c}+\mathbf{F}_{m o o r}+\mathbf{F}_{v i s}
$$

Here $\boldsymbol{\xi}=\left[\xi_{1} \ldots \xi_{6}\right]^{T}$ is the vector of displacements in the six DoFs, and a dot indicates time derivative. The frequency-domain tool WAMIT [12] was used to solve the radiation-diffraction problem (see Newman [37] and Lee [38]) and thus obtain the matrix of hydrostatic stiffness $\mathbf{C}_{h s t}$, as well as the frequencydependent matrices of added mass $\mathbf{A}(\omega)$ and radiation damping $\mathbf{B}_{\text {rad }}(\omega)$ and the frequency-dependent vector with the Fourier coefficients of the wave excitation loads, $\hat{\mathbf{F}}_{\text {exc }}(\omega)$. In Eqn. 11) $\mathbf{M}$ is the mass and inertia matrix, and $\mathbf{A}_{\infty}$ is the infinite-frequency limit of the added mass matrix. The wave excitation loads in the time domain are given by $\mathbf{F}_{e x c}$, while the mooring reactions and viscous effects are $\mathbf{F}_{\text {moor }}$ and $\mathbf{F}_{\text {vis }}$, respectively. The convolution integral in Eqn. (11) is due to radiation memory effects, where $\mathbf{K}(t)$ is the radiation-retardation kernel, given by the cosine transform of the radiation damping,

$$
\mathbf{K}(t)=\frac{2}{\pi} \int_{0}^{\infty} \mathbf{B}_{r a d}(\omega) \cos (\omega t) d \omega .
$$




\subsection{Simplification of viscous forcing and damping}

Viscous effects are commonly computed through the drag term of the Morison equation [39], which gives the drag force $d F$ normal to a cylindrical member section with diameter $D$ and length $d l$ as

$$
d F=\frac{1}{2} \rho C_{D} D\left|v_{f}-v_{s}\right|\left(v_{f}-v_{s}\right) d l .
$$

Here $\rho$ is the water density, $C_{D}$ is a drag coefficient, and $\left(v_{f}-v_{s}\right)$ is the local relative velocity between the fluid particles and the structure, perpendicular to the member axis. Equation 13 may also be written as

$d F=\frac{1}{2} \rho C_{D} D \operatorname{sgn}\left(v_{f}-v_{s}\right)\left(v_{f}-v_{s}\right)^{2} d l \equiv \frac{1}{2} \rho C_{D} D \operatorname{sgn}\left(v_{f}-v_{s}\right)\left(v_{f}^{2}+v_{s}^{2}-2 v_{f} v_{s}\right) d l$,

which shows that viscous effects are the sum of a pure forcing term, a quadratic damping term and a linear damping term proportional to the local fluid velocity. In Eqn. (11), the vector $\mathbf{F}_{v i s}$ corresponds to

$$
\mathbf{F}_{v i s}=\int_{l} \frac{1}{2} \rho C_{D} D\left|v_{f}-v_{s}\right|\left(v_{f}-v_{s}\right) d l .
$$

The structural velocity $v_{s}$ normal to the member axis at a location $\vec{r}$ is obtained by multiplying the local velocity by a normal unit vector $\vec{n}$,

$$
\left.v_{s}=\vec{n} \cdot\left[\begin{array}{lll}
\dot{\xi}_{1} & \dot{\xi_{2}} & \dot{\xi_{3}}
\end{array}\right]^{T}-\vec{r} \times\left[\begin{array}{lll}
\dot{\xi}_{4} & \dot{\xi_{5}} & \dot{\xi_{6}}
\end{array}\right]^{T}\right] .
$$

330

Under the assumption that $\operatorname{sgn}\left(v_{f}-v_{s}\right)=\operatorname{sgn}\left(v_{f}\right)$ and by neglection of the quadratic damping term, we approximate the drag loads as

$$
\mathbf{F}_{v i s} \approx \int_{l} \frac{1}{2} \rho C_{D} D\left|v_{f}\right| v_{f} d l-\int_{l} \rho C_{D} D\left|v_{f}\right| v_{s} d l \approx \mathbf{F}_{v i s^{\prime}}-\mathbf{B}_{v l} \dot{\boldsymbol{\xi}}
$$

where $\mathbf{F}_{v i s^{\prime}}$ is the integral of the drag forces considering the local pure fluid velocity (or the pure forcing term), and the linear damping term is written as a matrix applied to the bulk motion of the floater. As a further approximation $\mathbf{B}_{v l}$ is represented by a constant matrix. 
The main motivation for this simplification is that the overall linear damping will be calibrated later anyway. While the original relative Morison formulation will capture local phenomena better, the simpler damping format of Eqn. (17) is chosen due to ease of calibration and computational efficiency. The quadratic damping term, which is not considered here, dominates for decay tests in still water but it is expected to be less important than the linear term in wave conditions. Given the factor $\left|v_{f}\right|$ in the second term of Eqn. (17), $\mathbf{B}_{v l}$ is expected to increase in magnitude for increased wave velocity levels, for a successful approximation of Eqn. (15) by Eqn. (17). With the simplifications mentioned so far, a connection to a linear version of the EoM (solvable in the frequency domain) is also possible, although this step has not been taken in this study.

\subsection{Simplification of added mass and radiation damping}

Motivated also by the fact that OMA captures global linear damping ratios, the effect of the frequency-dependent radiation damping (represented by the convolution integral in Eqn. (11)) is also lumped into a constant linear global damping matrix $\mathbf{B}$, thus losing the frequency dependence. This step is justified by observing that generally all responses but heave are mainly dominated by resonance at the natural frequencies. For example, Fig. 3 shows the pitch diagonal element of the frequency-dependent radiation damping matrix, and the pitch diagonal element of the linear damping matrix after calibration for the sea state P1. It can be seen that the radiation damping alone is not able to counteract the resonance, given that its value at the natural frequency (black dashed line) is very small compared to the amount of damping needed to obtain a reasonable resonant response (red solid line). On the other hand, including both sources of damping leads to overdamping in the wave frequency range (green dashed lines), where the radiation damping is significantly higher than the constant linear damping. It is also worth noting that avoiding the convolution integral in the model significantly reduces the computational time.

Since the convolution integral also contributes to the added mass, the effect of its simplification on the response-amplitude operators (RAOs) was investi- 


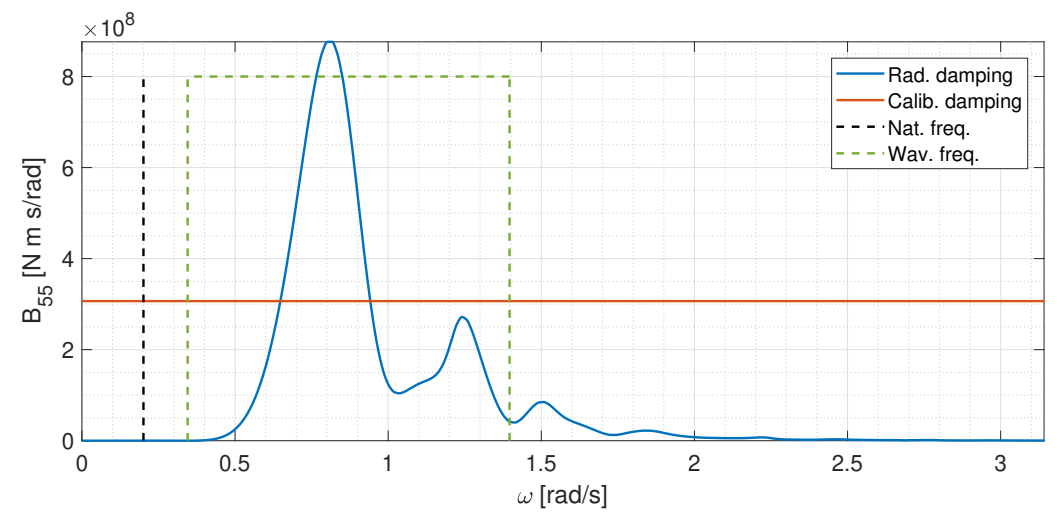

Figure 3: Radiation damping and the linear damping needed in the pitch degree of freedom for sea state P1. The black dashed line indicates the pitch natural frequency of the moored system, while the green dashed lines indicate the wave frequency range.

gated. As an example, the surge RAO for the moored floating wind turbine is shown in Fig. 4. It was computed from the first-order wave excitation loads and using a linearized mooring matrix. Three different RAOs are compared in the figure: the RAO using frequency-dependent added mass $\mathbf{A}(\omega)$ and radiation damping $\mathbf{B}_{\text {rad }}(\omega)$ from the WAMIT solution (blue); the RAO using the zero-frequency added mass $\mathbf{A}_{0}$ and the constant damping matrix $\mathbf{B}$ calibrated for sea state P1 (red); and the RAO using the infinite-frequency added mass $\mathbf{A}_{\infty}$ and the constant damping matrix $\mathbf{B}$ calibrated for sea state P1 (green). The left panel is centered at the surge natural frequency, and shows that the radiation damping alone would lead to an unrealistic surge response. The right panel focuses on the wave frequency range for the same sea state. Here we see that, while neglecting the convolution integral leads to errors in the RAO, the zero-frequency limit is a better alternative than the infinite-frequency limit in such case.

\subsection{The final equation of motion}

The modelling choices presented above mean that all the damping effects on the floater are lumped into the matrix $\mathbf{B}$, with the only exception being the viscous drag on the mooring system (see Section 5.8). The EoM implemented 

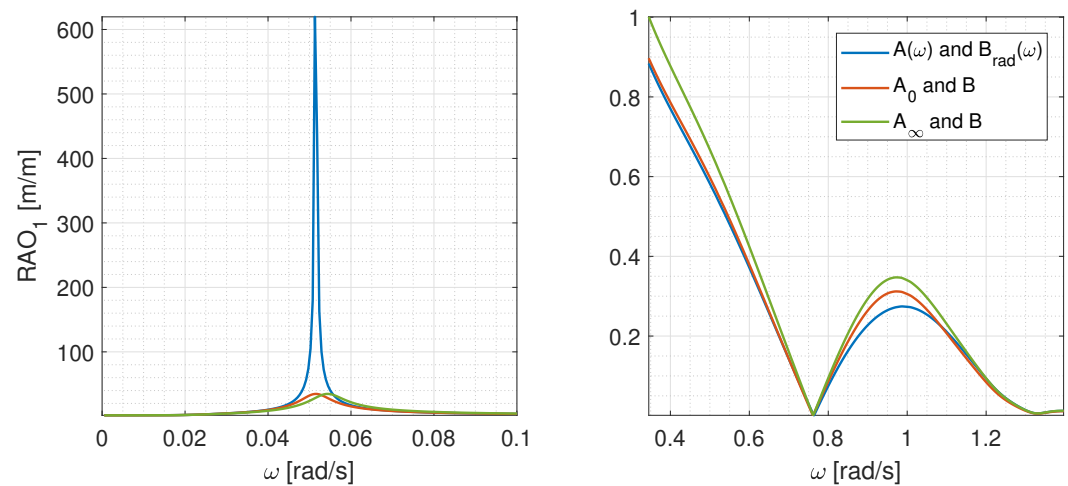

Figure 4: Comparison of surge response-amplitude operator using frequency-dependent and constant radiation properties. The left panel is centered at the surge natural frequency, while the right panel shows the wave frequency range for sea states P1 and P2.

in the model is therefore

$$
\left(\mathbf{M}+\mathbf{A}_{0}\right) \ddot{\boldsymbol{\xi}}+\mathbf{B} \dot{\boldsymbol{\xi}}+\mathbf{C}_{h s t} \boldsymbol{\xi}=\mathbf{F}_{e x c}+\mathbf{F}_{m o o r}+\mathbf{F}_{v i s^{\prime}}
$$
subsections.

\subsection{Linear damping matrix}

The linear damping matrix is build from damping ratios extracted from the test data with Operational Modal Analysis (see Section 2). For the present case, the input data array $\mathbf{x}(t)$ contained time series of measured surge, sway, heave, roll, pitch and yaw, hence $n c=6$. Each of the six raw signals was band-pass filtered with a bandwith of $0.03 \mathrm{~Hz}$ centered around the natural frequency of the given DoF. Furthermore, the correlation matrix $\mathbf{y}(t)$ was truncated at $t \leq 500$ $\mathrm{s}$, given that the correlation functions contained only noise for $t>500 \mathrm{~s}$.

395 Since OMA provides modal damping ratios, the linear damping matrix $\mathbf{B}$ is also built in the modal space. We choose to base the numerical modal damping matrix on the modal vectors of the numerical model, combined with the damping ratios from the OMA analysis. The numerical model's system matrix $\mathbf{D}_{\text {num }}$ is 
built as

$$
\mathbf{D}_{\text {num }}=\left(\mathbf{M}+\mathbf{A}_{0}\right)^{-1}\left(\mathbf{C}_{\text {hst }}+\mathbf{C}_{\text {moor }}\right),
$$

400 tion of the mooring system around the equilibrium position in still water. The diagonalization of $\mathbf{D}_{\text {num }}$ provides a set of eigenvectors contained in the columns of $\mathbf{\Psi}_{\text {num }}$, which is used to diagonalize the model's mass and stiffness matrices,

$$
\begin{array}{r}
{\left[m_{n}\right]=\boldsymbol{\Psi}_{\text {num }}^{T}\left(\mathbf{M}+\mathbf{A}_{0}\right) \boldsymbol{\Psi}_{\text {num }},} \\
{\left[k_{n}\right]=\mathbf{\Psi}_{\text {num }}^{T}\left(\mathbf{C}_{\text {hst }}+\mathbf{C}_{\text {moor }}\right) \boldsymbol{\Psi}_{\text {num }}}
\end{array}
$$

For each mode, the modal damping coefficient is thus computed as $b_{n}=$ $2 \zeta_{n} \sqrt{m_{n} k_{n}}$. Finally, the diagonal matrix of modal damping coefficients $\left[b_{n}\right]$ is transformed back to the physical space by virtue of

$$
\mathbf{B}=\left(\boldsymbol{\Psi}_{\text {num }}^{T}\right)^{-1}\left[b_{n}\right] \mathbf{\Psi}_{\text {num }}^{-1} .
$$

Given the findings of Ruzzo et al. [18, it is part of the present study to investigate how well the damping detected with OMA can lead to a good match between the measurements and the model output. For this reason, a calibrated modal damping ratio is also found for each sea state. The six modal damping ratios $\zeta_{n}$ are calibrated until the standard deviation $\sigma$ of the computed response in each DoF is as close as possible to the standard deviation of the measured response. In both signals the first $1080 \mathrm{~s}$ are discarded in the computation of $\sigma$. By working with modal damping coefficients, the damping in each DoF is independently calibrated with only one parameter.

\subsection{Wave excitation loads}

The vector of wave excitation loads contains first- and second-order components,

$$
\mathbf{F}_{e x c}=\mathbf{F}_{e x c}^{(1)}+\mathbf{F}_{e x c}^{(2)} .
$$


The first-order wave excitation loads for the $j^{\text {th }}$ DoF in the time domain are $X_{k, j}$ is the frequency-domain wave excitation load for a unit amplitude wave and $A_{k}$ is the wave amplitude. In the second-order problem, the loads arise from the interaction of wave pairs with unit amplitude and frequencies $\omega_{k}$ and $\omega_{l}$, producing loads at the difference frequency $\left(\omega_{k}-\omega_{l}\right)$ and at the sum frequency ${ }_{425}\left(\omega_{k}+\omega_{l}\right)$. These frequency-domain loads due to waves of unit amplitude are called quadratic transfer functions (QTFs), namely $Q T F_{k l, j}^{-}$for the difference frequencies and $Q T F_{k l, j}^{+}$for the sum frequencies. For an irregular sea state, the time series of second-order loads in the $j^{\text {th }}$ DoF is

$$
F_{e x c, j}^{(2)}=\Re\left\{\sum_{k=1}^{N} \sum_{l=1}^{N} A_{k} A_{l}^{*} Q T F_{k l, j}^{-} e^{i\left(\omega_{k}-\omega_{l}\right) t}+A_{k} A_{l} Q T F_{k l, j}^{+} e^{i\left(\omega_{k}+\omega_{l}\right) t}\right\}
$$

The double sum in Eqn. (24) can be computationally expensive if several frequencies are involved, therefore the symmetry in the QTF matrices is usually exploited. According to Pinkster [40, the second-order loads in Eqn. (24) are due to five components, namely:

1. the first-order relative wave elevation;

2. the quadratic interaction of the first-order velocity potential with itself;

3. the second-order velocity potential;

4. the interaction of the first-order potential and the first-order motion; and

5. the interaction of the first-order rotations with the first-order body accelerations.

As mentioned in 13, the first-order motion is sensitive to the amount of damping in the system. Since the viscous damping is expected to change with the sea state, the second-order analysis would have to be carried out for each sea state due to the dependence of the second-order solution on the first-order motion. Therefore, for the present work the contributions of the first-order motion to the second-order loads (items 4 and 5 in the list above) are not considered. ${ }_{445}$ This also allows a more general solution of the second-order problem, which is 
independent on the motion and can thus be re-used for different environmental conditions, including cases with wind forcing. This simplification may lead to an underprediction of the second-order loads for severe sea states. On the other hand, computation of the QTFs including the first-order solution with insufficient damping may lead to overestimation of the second-order loads [9].

\subsubsection{Computation and convergence of the second-order solution}

The time-domain implementation of the second-order hydrodynamic loads was verified against FAST v8 [5]. Full difference-frequency QTF matrices were computed in WAMIT for the six DoF, considering 50 angular frequencies in the range 0.0628-3.1416 rad/s, which corresponds to periods in the range 2100 s. This second-order analysis took almost 4 days, while the first-order WAMIT analysis was done for 200 frequencies and took $8 \mathrm{~h}$ and $30 \mathrm{~min}$. The sum-frequency loads were not included as they are expected to have negligible effects on the semisubmersible considered here (see [4, 9]). Taking advantage of symmetry, one quarter of the floater was discretized with 3719 panels (see Fig. 5 , right).
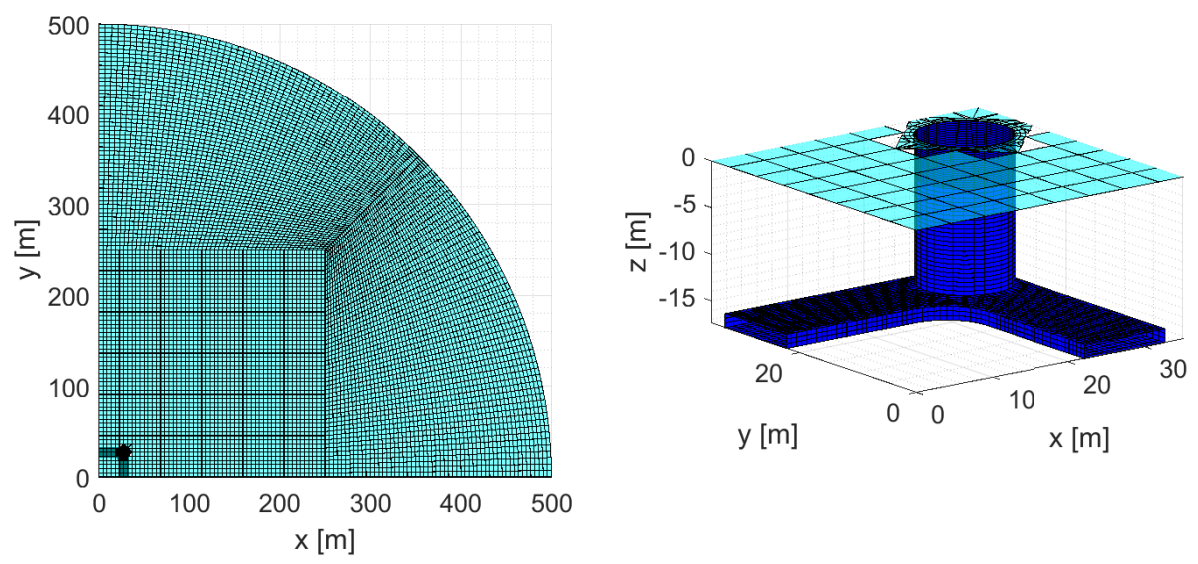

Figure 5: Panel discretization of the free surface (left) and the floater (right) for the WAMIT analysis.

The discretization of the free surface required a convergence study, where two parameters were varied: the radius of the partition inside which the integration 
of the quadratic forcing over the free surface is done numerically, RINNER; and the size of the free-surface panels relative to the body panel size at the free surface, SCALE. Results for RINNER values of 100, 300, 500 and 1000 $\mathrm{m}$ and SCALE values of 1,2, 4 and 8 were compared for angular frequencies corresponding to $1.00,1.05,1.10$ and $1.35 \mathrm{rad} / \mathrm{s}$ [41]. The convergence study suggested that, although the effect of the free surface was small, a discretization with RINNER=500 and SCALE=4 was appropriate (see Fig. 5. left). The agreement between the QTF results obtained with direct and indirect methods was also interpreted as a sign of numerical convergence with respect to panel resolution [1].

\subsection{Viscous drag forcing}

To compute the viscous drag forcing term $\mathbf{F}_{v i s^{\prime}}$, the floater was divided into four vertical columns and four rectangular horizontal members (square pontoon), as shown in Fig. 6. The geometry simplification adopted preserves the total area of the square pontoon, but leads to $2.76 \%$ error in the moment of area. The error in the computation of viscous drag forcing is expected to be of the same order of magnitude, and negligible in the overall dynamics due to the hydrodynamic loads being inertia-dominated.

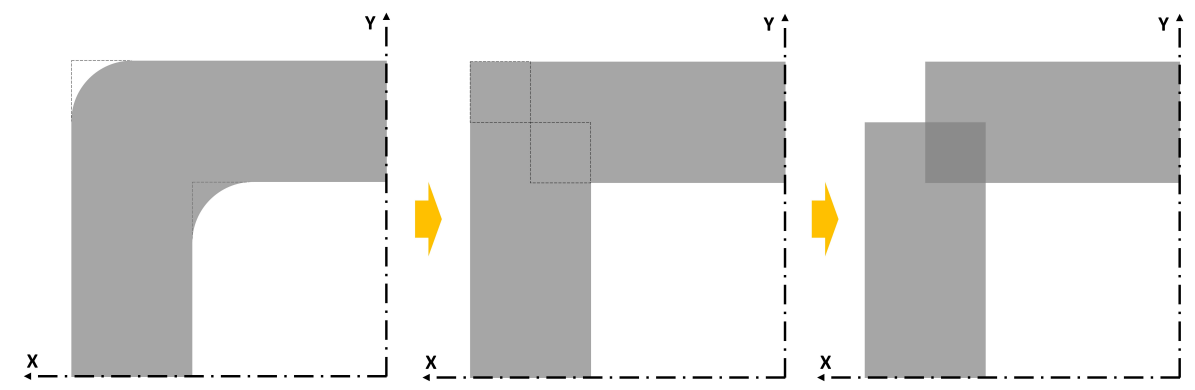

Figure 6: Simplification of the square pontoon geometry (bottom view) for calculating viscous drag loads on the NAUTILUS-10 floater.

Each of the eight members were discretized, and for each element the pure 
viscous drag forcing normal to the member axis was computed as

$$
d F=\frac{1}{2} \rho C_{D} D\left|v_{f}\right| v_{f} d l,
$$

where the wave particle velocity $v_{f}$ at each location was computed internally is built as

$$
\mathbf{M}=\left[\begin{array}{cccccc}
m & 0 & 0 & 0 & m z^{C M} & -m y^{C M} \\
& m & 0 & -m z^{C M} & 0 & m x^{C M} \\
& & m & m y^{C M} & -m x^{C M} & 0 \\
& & & I_{x}^{O} & -I_{x y}^{O} & -I_{z x}^{O} \\
& & & & I_{y}^{O} & -I_{y z}^{O} \\
& & & & & I_{z}^{O}
\end{array}\right]
$$

where $m$ is the total mass, $I_{x}^{O}, I_{y}^{O}$ and $I_{z}^{O}$ are the mass moments of inertia referred to the point of flotation $O,\left(x^{C M}, y^{C M}, z^{C M}\right)$ is the position of the centre of mass $(\mathrm{CM})$ and $I_{x y}^{O}, I_{y z}^{O}$ and $I_{z x}^{O}$ are the products of inertia. Note that due to symmetry in this case, $x^{C M}=y^{C M}=0$ and $I_{x y}^{O}=I_{y z}^{O}=I_{z x}^{O}=0$. All the non-zero values are taken as reported in [1].

In static equilibrium, the buoyancy force $\rho g V$ (with $V$ the displaced volume) is cancelled out by the sum of the total gravity force $m g$ and the net vertical force due to the mooring lines $F_{m o o r, z}$, hence these are not shown in the EoM. The hydrostatic restoring matrix $\mathbf{C}_{h s t}$ including contributions from waterplane and centre of buoyancy was imported from WAMIT, and the contribution from gravity was added internally in the model. 


\subsection{Mooring loads}

The mooring loads $\mathbf{F}_{\text {moor }}$ were computed externally by MoorDyn at each

505 time step and coupled to the model via a DLL. MoorDyn [34] is a dynamic lumped-mass mooring model that includes dynamic inertial loads, buoyancy and Morison-based hydrodynamic forces resulting from the motion of the lines in calm water (i.e. the mooring lines do not see the wave kinematics).

As mentioned above, even though the aim of this study is to have all damping effects lumped into the linear damping matrix, the drag forces on the mooring lines were enabled to avoid unphysical mooring line vibrations. The drag coefficient was estimated as $C_{D}=2$ based on the lab-scale Reynolds and KeuleganCarpenter numbers. The damping effect of mooring drag loads on the floater motion was quantified by carrying out decay simulations in the model with all the linear damping coefficients set to zero. Table 3 shows the linearized damping ratios estimated from these decay simulations, compared to the linearized damping ratios extracted from decay tests [1]. As observed in the table, the effect of mooring viscous drag on the floater motion is smaller for pitch and heave, while it is significant for surge and yaw. This will be taken into account when discussing the damping levels in the different operational sea states.

Table 3: Damping effects on the floater motion resulting from drag loads on the mooring lines, compared to the linearized damping ratios in the decay tests [1].

\begin{tabular}{lcccc}
\hline & Surge & Heave & Pitch & Yaw \\
\hline$\zeta_{\text {moor }}[\%]$ & 1.7 & 0.4 & 0.9 & 2.2 \\
$\zeta_{\text {test }}[\%]$ & 4.0 & 3.9 & 10.6 & 2.9 \\
\hline
\end{tabular}

\section{Results and discussion}

We now compare the numerical results with calibrated damping ratios to the test data for the four sea states shown in Tab. 2. To avoid a minor offset in the heave response, the displaced volume in the model was adjusted to $98.77 \%$ of 

tests, the initial value in each time series was subtracted for comparison to the numerical output. For each sea state, the response in each of the six DoFs is shown in terms of a representative portion of the time series (left column), a PSD plot (central column) and an exceedance probability plot (right column).

${ }_{530}$ For both the PSD and the exceedance probability analysis, the first $1080 \mathrm{~s}$ were discarded to avoid initial transient effects. The PSD signals were smoothened by applying a moving-average filter with 20 points. The red vertical dashed lines in the PSD plots indicate the natural frequency in each DoF reported in [1. The exceedance probability plots were obtained from peaks extracted from the in the sorted list. A minimum separation of $5 \mathrm{~s}$ between peaks was required To better compare the dynamics of the system, the mean was removed before carrying out the probability analysis. The mean responses are included in the plots of time series and are compared separately later in this section. The red

horizontal dashed lines in the probability plots correspond to the $95 \%$ percentile.

Each response plot is followed by a table comparing test and model response in each DoF in terms of the mean $\bar{\xi}$, standard deviation $\sigma$ and value at the $95 \%$ percentile $\xi^{95 \%}$. The relative error in $\sigma$ and $\xi^{95 \%}$ is also shown.

\subsection{Response to pink-noise waves}

Table 4: Results statistics for case P1.

\begin{tabular}{l|cc|ccc|ccc}
\hline & $\bar{\xi}_{\text {test }}$ & $\bar{\xi}_{\text {model }}$ & $\sigma_{\text {test }}$ & $\sigma_{\text {model }}$ & err.[\%] & $\xi_{\text {test }}^{95 \%}$ & $\xi_{\text {model }}^{95 \%}$ & err. [\%] \\
\hline Surge [m] & 0.40 & 0.54 & 0.69 & 0.69 & 0.0 & 1.46 & 1.55 & +6.2 \\
Sway [m] & -0.13 & -0.16 & 0.24 & 0.24 & 0.0 & 0.51 & 0.53 & +3.9 \\
Heave [m] & -0.02 & 0.01 & 0.12 & 0.12 & 0.0 & 0.29 & 0.31 & +6.9 \\
Roll [deg] & -0.01 & 0.00 & 0.04 & 0.04 & 0.0 & 0.11 & 0.10 & -9.1 \\
Pitch [deg] & 0.03 & -0.01 & 0.12 & 0.12 & 0.0 & 0.32 & 0.30 & -6.3 \\
Yaw [deg] & 0.04 & -0.03 & 0.21 & 0.17 & -19.0 & 0.50 & 0.45 & -10.0 \\
\hline
\end{tabular}



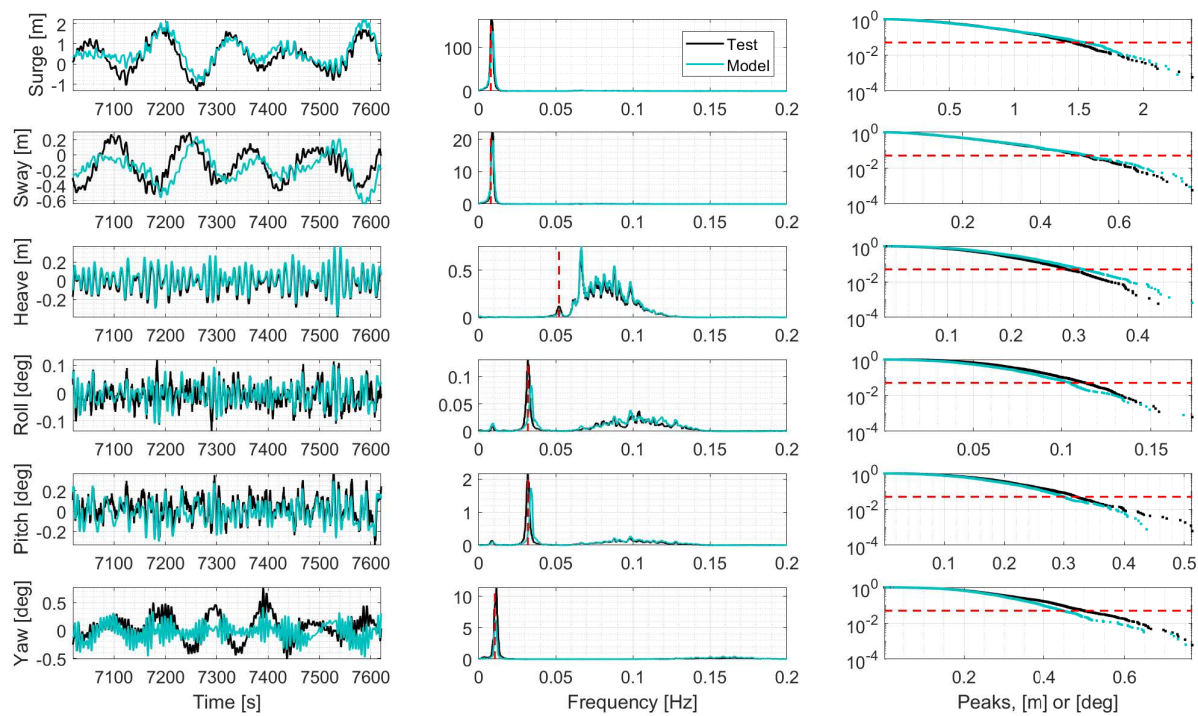

Figure 7: Time, frequency and exceedance probability plots for case P1.

The response to the pink-noise sea state $\mathrm{P} 1$ is shown in Fig. 7 and Tab. 4 All the DoFs but heave are dominated by resonance at the natural frequency, while the linear wave loads dominate the heave response and are also visible in roll and pitch. The model predicts well the motion in surge, sway and heave, with overpredictions up to $7 \%$ at the $95 \%$ percentile. The rotational motions show underpredictions up to $10 \%$, with the yaw motion also showing error in the standard deviation. It was observed that the yaw motion was underpredicted for all sea states, and a match of $\sigma_{6}$ was never possible even if the modal damping ratio $\zeta_{6}$ was set to zero. However, $\zeta_{6}=0$ does not mean that the yaw motion is completely undamped, since the drag on the mooring lines introduces significant damping in yaw, surge and sway (see Tab. 3). Thus, the yaw underprediction is believed to arise from missing wave excitation. This aspect is discussed in detail later.

Figure 8 and Tab. 5 show the response to the pink-noise sea state P2. The observations reported for P1 also apply to P2, although a better match of the 95\% percentile is obtained in this case, with errors below $5 \%$ in all DoFs but yaw. The yaw motion, on the other hand, is again significantly underpredicted 

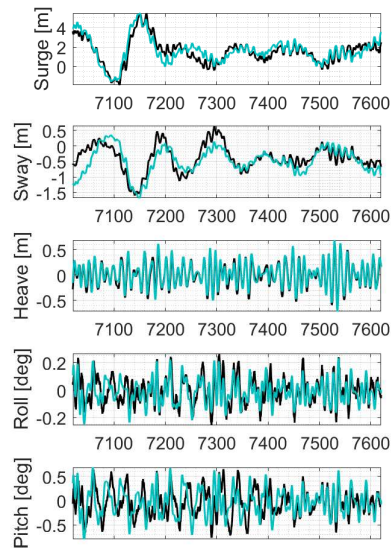

710072007300740075007600

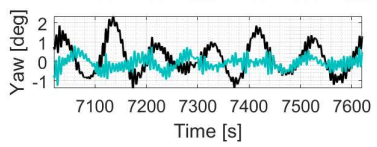

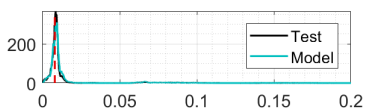
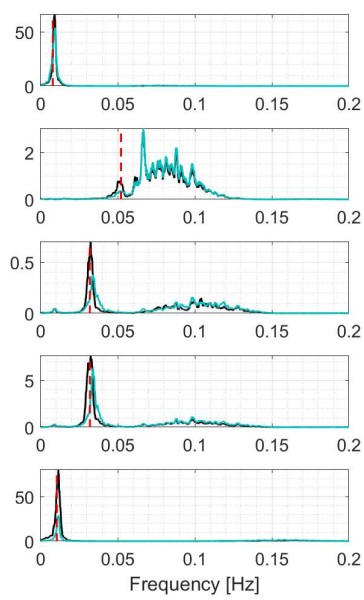
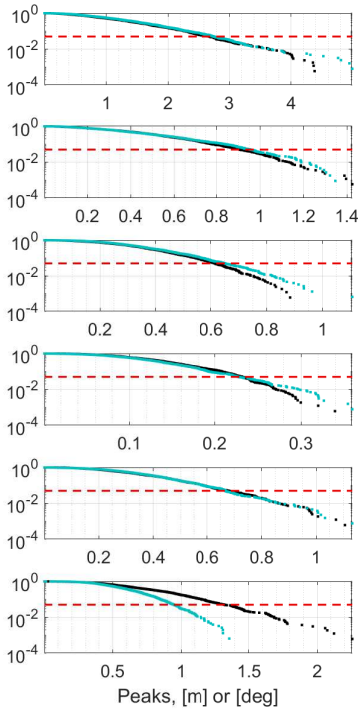

Figure 8: Time, frequency and exceedance probability plots for case P2.

Table 5: Results statistics for case P2

\begin{tabular}{l|cc|ccc|ccc}
\hline & $\bar{\xi}_{\text {test }}$ & $\bar{\xi}_{\text {model }}$ & $\sigma_{\text {test }}$ & $\sigma_{\text {model }}$ & err.[\%] & $\xi_{\text {test }}^{95 \%}$ & $\xi_{\text {model }}^{95 \%}$ & err.[\%] \\
\hline Surge [m] & 1.47 & 1.61 & 1.24 & 1.24 & 0.0 & 2.69 & 2.76 & +2.6 \\
Sway [m] & -0.35 & -0.49 & 0.45 & 0.45 & 0.0 & 0.91 & 0.95 & +4.4 \\
Heave [m] & -0.01 & 0.02 & 0.25 & 0.25 & 0.0 & 0.62 & 0.65 & +4.8 \\
Roll [deg] & 0.00 & -0.01 & 0.09 & 0.09 & 0.0 & 0.23 & 0.23 & 0.0 \\
Pitch [deg] & -0.04 & -0.04 & 0.26 & 0.26 & 0.0 & 0.68 & 0.67 & -1.5 \\
Yaw [deg] & 0.08 & -0.09 & 0.57 & 0.38 & -33.3 & 1.32 & 0.95 & -28.0 \\
\hline
\end{tabular}

with errors larger than those reported for $\mathrm{P} 1$.

\subsection{Response to Pierson-Moskowitz waves}

The response to the Pierson-Moskowitz sea state M1 is shown in Fig. 9 and

Tab. 6. Surge, sway and yaw are again dominated by resonance, while heave is dominated by linear wave loads and roll and pitch show similar response levels at the wave range and at the natural frequency. Although a good match is observed in the exceedance probability plots, the spectral comparison seems less 

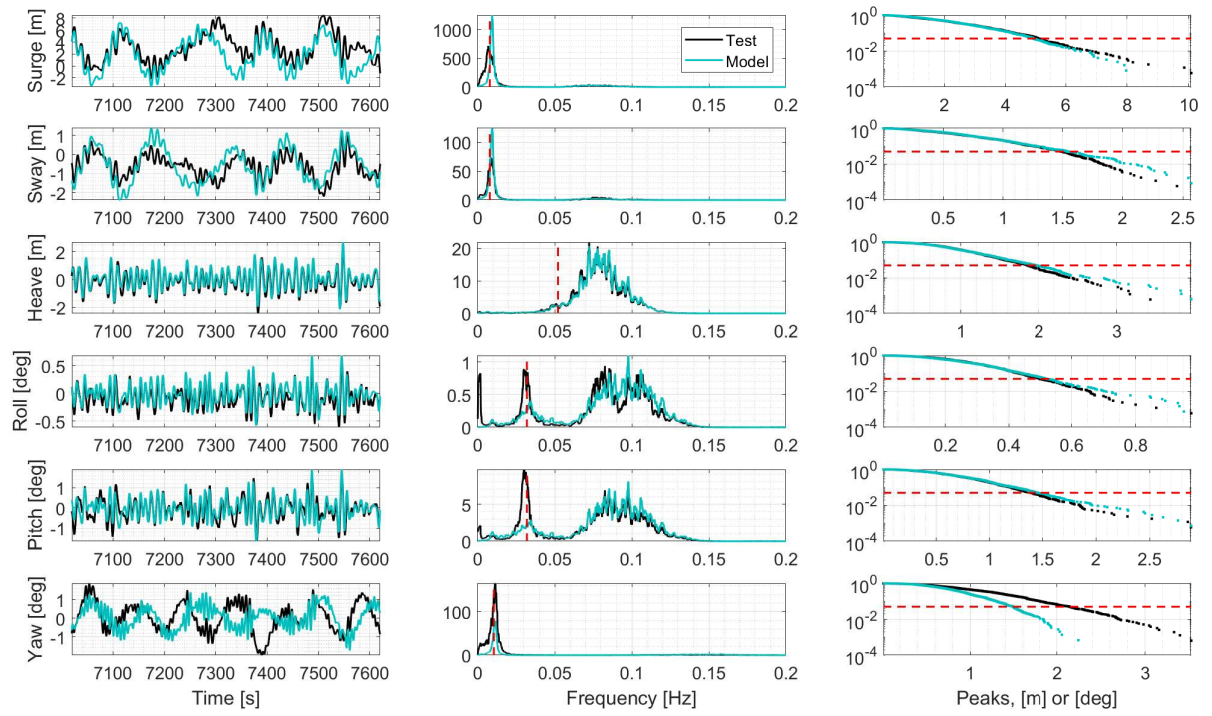

Figure 9: Time, frequency and exceedance probability plots for case M1.

Table 6: Results statistics for case M1.

\begin{tabular}{l|cc|ccc|ccc}
\hline & $\bar{\xi}_{\text {test }}$ & $\bar{\xi}_{\text {model }}$ & $\sigma_{\text {test }}$ & $\sigma_{\text {model }}$ & err.[\%] & $\xi_{\text {test }}^{95 \%}$ & $\xi_{\text {model }}^{95 \%}$ & err. [\%] \\
\hline Surge [m] & 3.03 & 1.86 & 2.38 & 2.14 & -10.1 & 5.09 & 4.92 & -3.3 \\
Sway [m] & -0.64 & -0.54 & 0.68 & 0.68 & 0.0 & 1.49 & 1.54 & +3.4 \\
Heave [m] & -0.10 & 0.06 & 0.73 & 0.73 & 0.0 & 1.85 & 1.96 & +5.9 \\
Roll [deg] & -0.11 & -0.01 & 0.19 & 0.19 & 0.0 & 0.50 & 0.52 & +4.0 \\
Pitch [deg] & -0.09 & -0.05 & 0.53 & 0.53 & 0.0 & 1.39 & 1.45 & +4.3 \\
Yaw [deg] & 0.00 & -0.02 & 0.96 & 0.59 & -38.5 & 2.15 & 1.49 & -30.7 \\
\hline
\end{tabular}

good than for the pink-noise cases discussed above, especially for roll and pitch.

This is a consequence of the calibration criterion, since the standard deviation $\sigma$ relates to the response spectrum through $\sigma_{\xi, j}^{2}=\int_{0}^{\infty} S_{\xi, j}(\omega) d \omega$. Hence, matching $\sigma$ ensures that the area under the response spectrum between test and model is the same, although local differences may exist. The $95 \%$ percentile values deviate up to $6 \%$, with the yaw motion showing a worse prediction than for $\mathrm{P} 1$ and P2. The standard deviation of the surge response could not be matched in 
this case, even with $\zeta_{1}=0$. Still, a good match is observed in the exceedance probability plot and the $95 \%$ percentile. As discussed for the yaw motion earlier, the drag on the mooring lines introduces significant damping in the horizontal DoFs, thus this level of damping combined with missing wave excitation forces leads to a mismatch in $\sigma_{1}$.
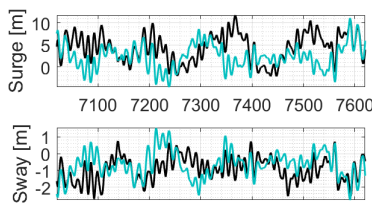

710072007300740075007600
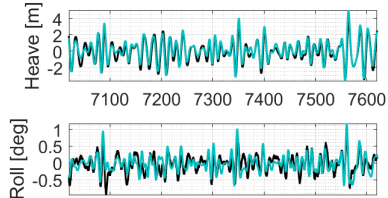

710072007300740075007600

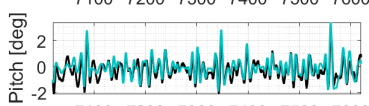

$7100 \quad 72007300740075007600$

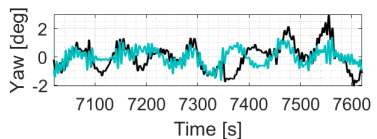

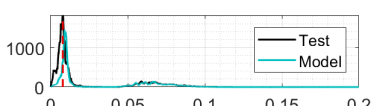
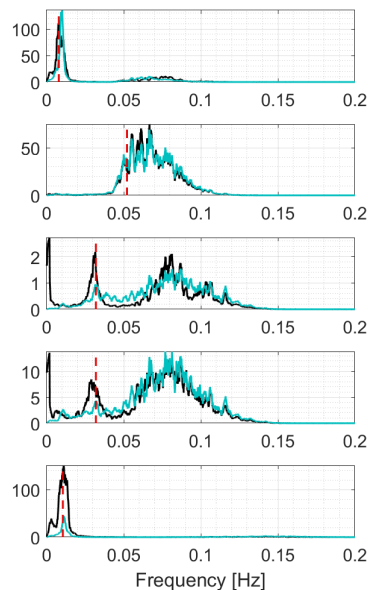
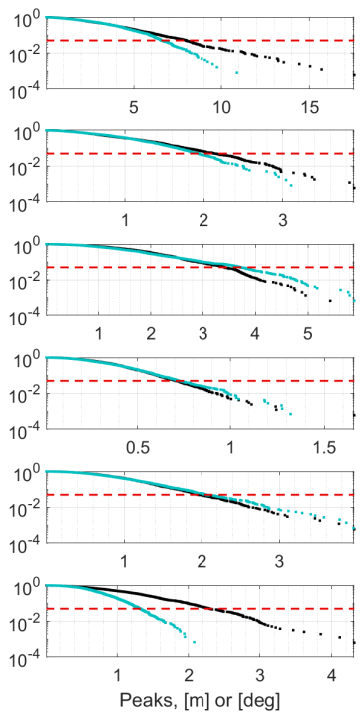

Figure 10: Time, frequency and exceedance probability plots for case M2.

Table 7: Results statistics for case M2

\begin{tabular}{l|cc|ccc|ccc}
\hline & $\bar{\xi}_{\text {test }}$ & $\bar{\xi}_{\text {model }}$ & $\sigma_{\text {test }}$ & $\sigma_{\text {model }}$ & err.[\%] & $\xi_{\text {test }}^{95 \%}$ & $\xi_{\text {model }}^{95 \%}$ & err.[\%] \\
\hline Surge [m] & 4.05 & 1.99 & 3.64 & 2.92 & -19.8 & 7.97 & 6.59 & -17.3 \\
Sway [m] & -0.80 & -0.58 & 0.95 & 0.84 & -11.6 & 2.13 & 1.93 & -9.4 \\
Heave [m] & -0.14 & 0.08 & 1.36 & 1.36 & 0.0 & 3.41 & 3.72 & +9.1 \\
Roll [deg] & -0.06 & -0.01 & 0.27 & 0.27 & 0.0 & 0.71 & 0.72 & +1.4 \\
Pitch [deg] & -0.19 & -0.04 & 0.76 & 0.76 & 0.0 & 1.95 & 2.06 & +5.6 \\
Yaw [deg] & 0.03 & -0.04 & 1.05 & 0.52 & -50.5 & 2.29 & 1.32 & -42.4 \\
\hline
\end{tabular}

Figure 10 and Tab. 7 show the response to the Pierson-Moskowitz sea state M2. The observations reported for M1 also apply to M2, with errors within $10 \%$ 
at the $95 \%$ percentile for sway, heave, roll and pitch. The yaw prediction keeps getting worse with increasing sea state severity, with errors in $\sigma_{6}$ above $50 \%$. In this case, although $\zeta_{1}=\zeta_{2}=\zeta_{6}=0$ the standard deviation of surge, sway and yaw could not be matched, likely due to the combination of mooring drag damping and missing wave excitation. It is believed that this underprediction of wave excitation loads arises from the simplification of the QTFs (see Section 5.5), where the effect of the first-order motion on the second-order solution was neglected. The underprediction becomes more apparent as the sea state severity increases, given that the relative magnitude of the second-order loads to the firstorder loads increases with the wave height. This effect is most pronounced in the horizontal DoFs (surge, sway and yaw), because these are always dominated by resonance and thus are more sensitive to the prediction of second-order loads. Heave is always dominated by linear wave loads, and roll and pitch are only dominated by resonance for mild sea states (P1 and P2), whereas for M1 and M2 they are also largely influenced by linear wave loads.

\subsection{Mean-drift response}

Figure 11 shows the measured and modelled mean-drift responses in surge and sway for the four sea states (see Tabs. 44-7). The predictions are slightly overestimated for the mild sea states P1 and P2, while considerably underestimated for the sea states M1 and M2 (especially in surge, where the error is $-51 \%)$. This underprediction is likely due to the missing second-order wave excitation loads associated to the simplification in the QTFs, as well as to the viscous drag forces being integrated only up to $z=0$ (see Section 5.6). Viscous mean drift is a third-order effect that occurs when the viscous forces are integrated up to the instantaneous free surface, or when they are applied to the instantaneous floater position (see Faltinsen 43]). The lack of these third-order effects in the model may also contribute to the underprediction of mean-drift 

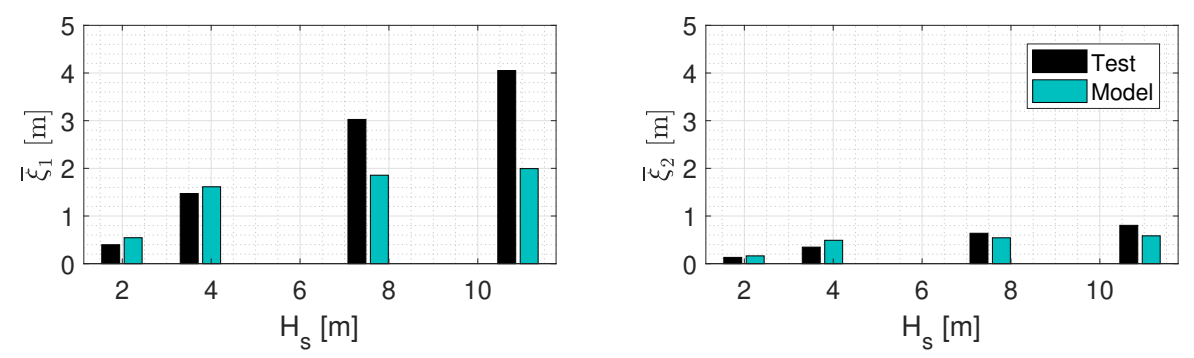

Figure 11: Mean-drift surge and sway responses for different sea states.

\subsection{Comparison of OMA and calibrated damping}

Figure 12 shows the damping ratios obtained by calibration and OMA analysis as a function of significant wave height.
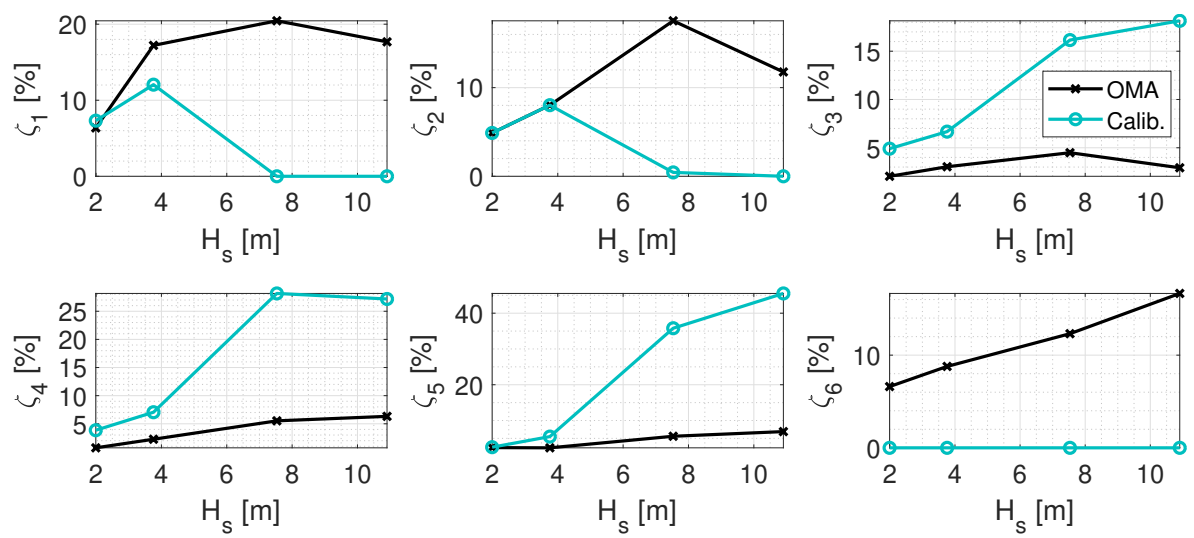

Figure 12: Damping ratios for different sea states, estimated with OMA and calibrated.

The damping ratios estimated with OMA (black) generally increase with

$H_{s}$, as reported in Sall [19. The increasing trend is clear for all modes but yaw up to M1, but a drop between the last two sea states is seen for surge, sway and heave. This observation can be linked to the assumptions OMA works under: i) linear system; ii) lightly-damped motions (10-15\% damping); and iii) white-noise excitation loads. The three assumptions are likely to be fulfilled for the pinknoise mild sea states (P1 and P2). It is also expected that similar results can be obtained in white-noise mild sea states. For the Pierson-Moskowitz spectra 
(M1 and M2), however, assumption (iii) may only be fulfilled if the wave peak frequency is far enough from the system natural frequencies, which is more likely to be true for M1 than for M2. This is consistent with the observations reported in Ruzzo et al. 18. Further, for case M2 (50-year sea state) the system is less likely to behave in a linear manner, thus (i) may also be violated. In other words, OMA may not be able to reliably detect the damping levels for M1 and M2. In addition, Bajrić et al. 17 reported that the damping estimated by OMA may not be reliable when the system has closely-spaced modes. This may also play a role in this case, since the surge-sway and pitch-roll DoFs have the same natural frequencies due to symmetry.

The calibrated damping ratios (blue) are close to the OMA predictions for case $\mathrm{P} 1$. The prediction is worse for P2, and completely deviates for M1 and M2 in all DoFs. The differences between the OMA-predicted and calibrated damping ratios are likely due to three different reasons: i) the OMA assumpions and how they are less likely to be fulfilled for the sea states M1 and M2; ii) the missing wave excitation; and iii) the damping introduced by the mooring lines. As discussed above, neglecting the first-order motion in the computation of the QTFs leads to underprediction of the second-order loads for severe sea states, and its effect is more visible in the DoFs that are dominated by resonance namely surge, sway and yaw. These are also the DoFs where the damping introduced by the mooring lines is relatively larger (see Tab. 3). In a dynamic system, the same level of response can be maintaned if both excitation and damping are reduced. In the model, the missing second-order wave loads and the mooring damping for M1 and M2 are compensated by a drop in the calibrated damping level, as seen for surge and sway in the first two panels of Fig. 12 . The yaw DoF is missing excitation in all sea states, hence the best possible match is obtained with $\zeta_{6}=0$, which still leads to underpredicted motion due to the damping effect of the mooring lines.

${ }_{650}$ We note that in operational cases with wind the aerodynamic loads (which introduce both forcing and damping) are expected to dominate the low-frequency motion (see, for example, [10]), and may also change the applicability of OMA 
for estimation of damping properties.

\subsection{Comparison to damping calibrated with decay tests}

Often the decay tests are used to calibrate linear and quadratic damping matrices in the numerical models. Decay tests in still water only provide response information at the system natural frequencies and with no waves, where the quadratic damping mechanism dominates. On the other hand, the response to waves is generally dominated by the linear damping mechanism, which is sea-state dependent. Thus we here investigate how well this decay-calibration method works within the present model. With this purpose a quadratic damping matrix was introduced, and the diagonal values of linear and quadratic damping matrices were calibrated to match the decay tests, as shown in Fig. 13. For each pair of consecutive peaks in the decay signals, a damping ratio was computed from the logarithmic decrement and associated to the mean amplitude between the two peaks.
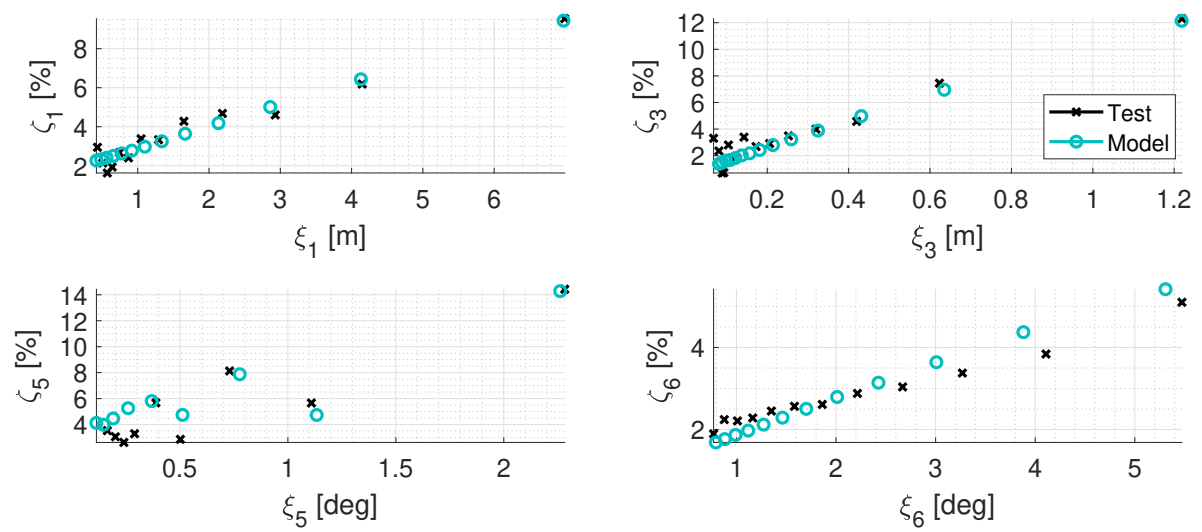

Figure 13: Damping ratios as a function of amplitude extracted from decay tests and simulations.

The results for case $\mathrm{P} 1$ and the damping calibrated with the decay tests are shown in Fig. 14 and Tab. 8. When compared to the results for the same case with the calibrated modal damping ratios (Fig. 7 and Tab. 44), it is seen that the prediction is worse for all DoFs. This illustrates that inclusion 
of a decay-calibrated, linear-quadratic damping format is not sufficient for the present model. An extended investigation for a model with the complete relative Morison formulation is left as a relevant future investigation.
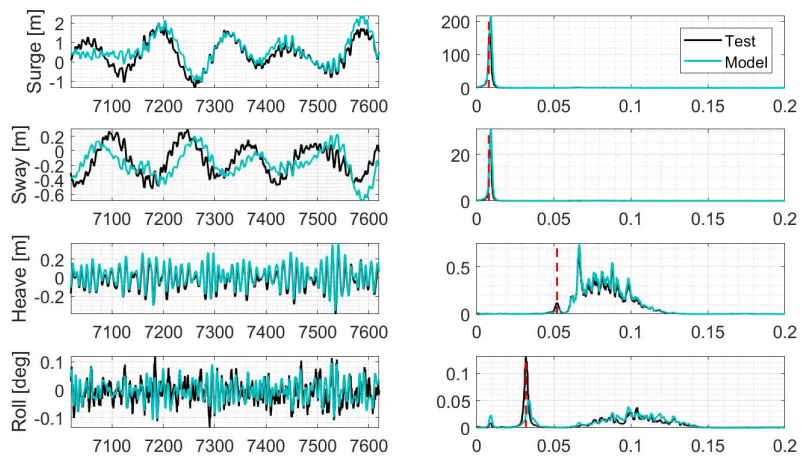

\begin{tabular}{rrrr|}
710072007300740075007600 \\
कृ
\end{tabular}

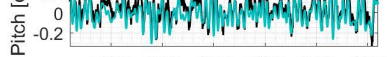

710072007300740075007600
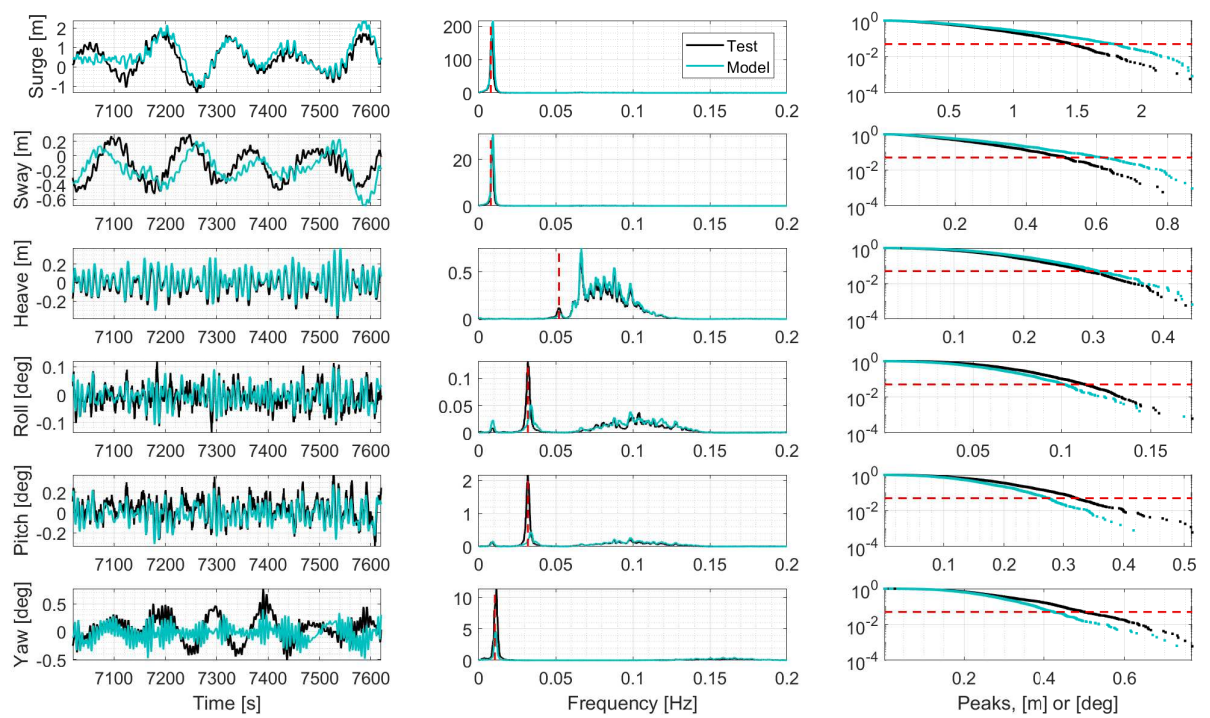

Figure 14: Time, frequency and exceedance probability plots for case P1 with damping calibrated to decay tests.

Table 8: Results statistics for case P1 with damping calibrated to decay tests.

\begin{tabular}{l|cc|ccc|ccc}
\hline & $\bar{\xi}_{\text {test }}$ & $\bar{\xi}_{\text {model }}$ & $\sigma_{\text {test }}$ & $\sigma_{\text {model }}$ & err.[\%] & $\xi_{\text {test }}^{95 \%}$ & $\xi_{\text {model }}^{95 \%}$ & err.[\%] \\
\hline Surge [m] & 0.40 & 0.54 & 0.69 & 0.80 & +15.9 & 1.46 & 1.80 & +23.3 \\
Sway [m] & -0.13 & -0.16 & 0.24 & 0.28 & +16.7 & 0.51 & 0.62 & +21.6 \\
Heave [m] & -0.02 & 0.01 & 0.12 & 0.12 & 0.0 & 0.29 & 0.31 & +6.9 \\
Roll [deg] & -0.01 & 0.00 & 0.04 & 0.04 & 0.0 & 0.11 & 0.10 & -9.1 \\
Pitch [deg] & 0.03 & -0.01 & 0.12 & 0.10 & -16.7 & 0.32 & 0.28 & -12.5 \\
Yaw [deg] & 0.04 & -0.03 & 0.21 & 0.16 & -23.8 & 0.50 & 0.43 & -14.0 \\
\hline
\end{tabular}




\section{Conclusions}

675

Slow-drift forcing and damping are important for an accurate reproduction of the low-frequency motion of floating offshore wind turbines. Here we developed a numerical model that includes both inviscid slow-drift forcing through full QTF and viscous forcing. The model was set up to accommodate a linear damping matrix that represents all the damping effects on the floater, except the damping from the external mooring line model. For that purpose, the classical Morison formulation of the drag forcing with relative velocity was separated into a pure forcing term and an approximate constant linear damping term, which can be calibrated to match the experiments. The linear damping representation enables a linear response formulation, which is attractive due to the easy calibration and later numerical speed-up possibilities. Further, in the interest of numerical efficiency and with little error, the frequency-dependent radiation properties were replaced by constant added mass and damping matrices. As a novel feature, the damping ratios were calibrated in the modal space, and later transformed to the physical space before applying them in the model. The linear damping matrix was calibrated for each sea state to fit the standard deviation of the measured response. Next, we investigated the possibilities of applying OMA to estimate the damping ratios.

The model was validated with wave basin data for four sea states with increasing severity, including the 50-year sea state. All the cases considered included waves misaligned with the structure's axes of symmetry, hence inducing three-dimensional response. Comparison to test data was made in terms of time series, PSD, and exceedance probability plots.

For the operational sea state with $H_{s}=7.7 \mathrm{~m}$, the damping calibration method of matching the standard deviation of the response gave quite good results with the surge motion being within $3.3 \%$ at the $95 \%$ quantile level. Similarly, heave and pitch was reproduced with a relative error of $5.9 \%$ and $4.3 \%$ respectively at the $95 \%$ level. The yaw response was always underpredicted, since even zero damping added in yaw implied an underprection for this DoF. 
This underprediction also occurred in the 50-year sea state of $H_{s}=10.9 \mathrm{~m}$, QTFs found in the literature, such as the "white-noise approach". This will enable faster evaluation of the slow-drift response of floating wind turbines. 


\section{Acknowledgements}

used for this study. Sintef Ocean (www.sintef.no/en/ocean) is also thanked for providing access to the experimental data.

\section{References}

[1] M. Thys, V. Chabaud, T. Sauder, L. Eliassen, L. Sæther, Ø. Magnussen,

[4] T. Duarte, A. Sarmento, J. Jonkman, Effects of second-order hydrodynamic forces on floating offshore wind turbines, in: Proceedings of the 32nd ASME Wind Energy Symposium, 2014. doi:10.2514/6.2014-0361.

[5] J. Jonkman, B. Jonkman, NWTC Information Portal (FAST v8) (2016). Real-time hybrid model testing of a semi-submersible 10MW floating wind turbine and advances in the test method, in: Proceedings of the ASME 1st International Offshore Wind Technical Conference (IOWTC 2018), 2018. doi:10.1115/IOWTC2018-1081

[2] L. Roald, J. Jonkman, A. Robertson, N. Chokani, The effect of secondorder hydrodynamics on floating offshore wind turbines, Energy Procedia 35 (2013) 253-264. doi:10.1016/j.egypro.2013.07.178

[3] I. Bayati, J. Jonkman, A. Robertson, A. Platt, The effects of second-order hydrodynamics on a semisubmersible floating offshore wind turbine, Journal of Physics: Conference Series 524. doi:10.1088/1742-6596/524/1/ 012094
URL https://nwtc.nrel.gov/FAST8 
[6] Orcina, OrcaFlex

URL https://www. orcina.com/SoftwareProducts/OrcaFlex/

[7] J. Newman, Second-order, slowly-varying forces on vessels in irregular waves, in: Proceedings of the Symposium on the Dynamics of Marine Vehicles and Structures in Waves, London, 1974, pp. 182-186.

[8] R. Standing, W. Brendling, D. Wilson, Recent developments in the analysis of wave drift forces, low-frequency damping and response, in: Offshore Technology Conference, Houston, Texas, 1987. doi:10.4043/5456-MS

[9] S. Gueydon, T. Duarte, J. Jonkman, I. Bayati, A. Sarmento, Comparison of second-order loads on a semisubmersible floating wind turbine, in: Proceedings of the ASME 33rd International Conference on Ocean, Offshore and Arctic Engineering (OMAE 2014), 2014. doi:10.1115/OMAE2014-23398.

[10] A. Coulling, A. Goupee, A. Robertson, J. Jonkman, Importance of secondorder difference-frequency wave-diffraction forces in the validation of a FAST semi-submersible floating wind turbine model, in: Proceedings of the ASME 32nd International Conference on Ocean, Offshore and Arctic Engineering (OMAE 2013), 2013. doi:10.1115/OMAE2013-10308.

[11] V. Matos, A. Simos, S. Sphaier, Second-order resonant heave, roll and pitch motions of a deep-draft semi-submersible: Theoretical and experimental results, Ocean Engineering 38 (2011) 2227-2243. doi:10.1016/j . oceaneng. 2011.10 .005

[12] C. Lee, J. Newman, WAMIT (2016).

URL http://www. wamit.com/

[13] A. Simos, F. Ruggeri, R. Watai, A. Souto-Iglesias, C. Lopez-Pavon, Slowdrift of a floating wind turbine: An assessment of frequency-domain methods based on model tests, Renewable Energy 116 (2018) 133-154. doi:10.1016/j.renene.2017.09.059. 
[14] F. Lemmer, W. Yu, P. Cheng, A. Pegalajar-Jurado, M. Borg, R. Mikkelsen, H. Bredmose, The Triple Spar campaign: Validation of a reduced-order simulation model for floating wind turbines, in: Proceedings of the ASME 37th International Conference on Ocean, Offshore and Arctic Engineering (OMAE 2018), 2018. doi:10.1115/OMAE2018-78119.

[15] M. Kvittem, P. Berthelsen, L. Eliassen, M. Thys, Calibration of hydrodynamic coefficients for a semi-submersible 10MW wind turbine, in: Proceedings of the ASME 37th International Conference on Ocean, Offshore and Arctic Engineering (OMAE 2018), 2018. doi:10.1115/OMAE2018-77826.

[16] T. Pahn, R. Rolfes, J. Jonkman, Inverse load calculation procedure for offshore wind turbines and application to a 5-MW wind turbine support structure, Wind Energy 20 (2017) 1171-1186. doi:10.1002/we.2088.

[17] A. Bajrić, J. Høgsberg, F. Rüdinger, Evaluation of damping estimates by automated Operational Modal Analysis for offshore wind turbine tower vibrations, Renewable Energy 116 (2018) 153-163. doi:10.1016/j.renene. 2017.03 .043

[18] C. Ruzzo, G. Failla, M. Collu, V. Nava, V. Fiamma, F. Arena, Operational Modal Analysis of a spar-type floating platform using Frequency Domain Decomposition method, Energies 9. doi:10.3390/en9110870.

[19] P. W. Sall, Modal identification of damping for floating wind turbines. MSc thesis, DTU. (2017).

[20] H. Bredmose, F. Lemmer, M. Borg, A. Pegalajar-Jurado, R. Mikkelsen, T. Larsen, T. Fjelstrup, W. Yu, A. Lomholt, L. Boehm, J. AzconaArmendariz, The Triple Spar campaign: Model tests of a 10MW floating wind turbine with waves, wind and pitch control, Energy Procedia 137 (2017) 58-76. doi:10.1016/j.egypro.2017.10.334.

[21] C. Ruzzo, G. Failla, M. Collu, V. Nava, V. Fiamma, F. Arena, Output-only identification of rigid-body motions of floating structures: a case study, 
Procedia Engineering 199 (2017) 930-935. doi:10.1016/j.proeng. 2017. 09.243

[22] V. Nava, J. Galvan-Fernandez, M. Sanchez-Lara, C. Garrido-Mendoza, G. Perez-Moran, M. Le Boulluec, B. Augier, R. Rodriguez-Arias, Hydrodynamic identification of NAUTILUS FOWT platform from small-scale tests, in: Proceedings of the 3rd International Conference on Renewable Energies Offshore (RENEW 2018), 2018.

[23] R. Brincker, C. Ventura, Introduction to Operational Modal Analysis, 1st Edition, Wiley, 2015.

825 [24] S. Ibrahim, E. Mikulcik, A method for direct identification of vibration parameters from the free response, The Shock and Vibration Bulletin 47 (1977) 183-196.

[25] S. Ibrahim, Random decrement technique for modal identification of structures, Journal of Spacecraft and Rockets 14 (11) (1977) 696-700.

[26] S. Ibrahim, Modal confidence factor in vibration testing, Journal of Spacecraft and Rockets 15 (5) (1978) 313-316.

[27] C. Bak, F. Zahle, R. Bitsche, T. Kim, A. Yde, L. Henriksen, A. Natarajan, M. Hansen, Description of the DTU 10 MW reference wind turbine, Tech. rep., No. I-0092, DTU Wind Energy (2013).

835 [28] J. Galvan-Fernandez, M. Sanchez-Lara, I. Mendikoa-Alonso, V. Nava, F. Boscolo-Papo, C. Garrido-Mendoza, J. Berque, G. Perez-Moran, R. Rodriguez-Arias, Definition and analysis of NAUTILUS-DTU10 MW floating offshore wind turbine at Gulf of Maine. Experiments at Sintef Ocean and PoliMi, Tech. rep., Tecnalia R\&I (2018).

${ }_{840}[29]$ W. Yu, K. Müller, F. Lemmer, LIFES50+ D4.2: Public definition of the two LIFES50+ 10MW floater concepts, Tech. rep., University of Stuttgart (2018). 
[30] M. Thys, LIFES50+ D3.4: Ocean basin test report, Tech. rep., Sintef Ocean (2018).

${ }_{845}$ [31] T. Sauder, V. Chabaud, M. Thys, E. Bachynski, L. Sæther, Real-time hybrid model testing of a braceless semi-submersible wind turbine. Part I: the hybrid approach, in: Proceedings of the ASME 35th International Conference on Ocean, Offshore and Arctic Engineering (OMAE 2016), 2016. doi:10.1115/OMAE2016-54435

[32] V. Chabaud, L. Eliassen, M. Thys, T. Sauder, Multiple-degree-of-freedom actuation of rotor loads in model testing of floating wind turbines using cable-driven parallel robots, Journal of Physics: Conference Series 1104 (1) (2018) 012021. doi:10.1088/1742-6596/1104/1/012021.

[33] A. Krieger, G. Ramachandran, L. Vita, G. Gómez-Alonso, J. Berque, G. Aguirre, LIFES50+ D7.2: Design basis, Tech. rep., DNV-GL (2015).

[34] M. Hall, MoorDyn (2017). URL http://www.matt-hall.ca/moordyn.html

[35] W. Cummins, The impulse response functions and ship motions, Schiffstechnik 9 (1962) 101-109. doi:10.1.1.839.7678.

[36] T. Ogilvie, Recent progress toward the understanding and prediction of ship motions, in: Fifth Symposium on Naval Hydrodynamics, 1964, pp. $3-128$.

[37] J. Newman, Marine hydrodynamics, 1st Edition, The MIT Press, 1977.

[38] C. Lee, WAMIT Theory Manual, Tech. rep., No. 95-2, Massachusetts Institute of Technology, Cambridge, Massachusetts (1995).

[39] J. Morison, J. Johnson, S. Schaaf, The force exerted by surface waves on 口 piles, Journal of Petroleum Technology 2 (05) (1950) 149-154. doi:10. 2118/950149-G 
[40] J. Pinkster, Low-frequency second-order wave exciting forces on floating structures, Ph.D. thesis, Technical University of Delft (1980).

[41] L. Roald, J. Jonkman, A. Robertson, The effect of second-order hydrodynamics on a floating offshore wind turbine, Tech. rep., No. NREL/TP5000-61452, National Renewable Energy Laboratory (2014).

[42] G. Airy, Tides and waves (1845).

875 [43] O. Faltinsen, Sea loads on ships and offshore structures, 1st Edition, Cambridge University Press, 1990. 